\title{
Spontaneous Deceleration and Acceleration of Growth Rate in Medullary Thyroid Carcinomas Suggested by Changes in Calcitonin Doubling Times Over Long-Term Surveillance
}

\author{
Akira Miyauchi $^{1} \cdot$ Takumi Kudo $^{2} \cdot$ Minoru Kihara $^{1} \cdot$ Hitomi Oda $^{1} \cdot$ Yasuhiro Ito $^{1} \cdot$ \\ Akihiro Miya ${ }^{1}$
}

Published online: 18 September 2018

(C) The Author(s) 2018

\begin{abstract}
Background Based on our long-term observation of medullary thyroid carcinoma (MTC) patients, we hypothesized that some MTCs have spontaneous deceleration or regression of tumor growth over a long term and that a minority may acquire growth acceleration. We thus compared the calcitonin doubling time (Ct-DT) in the earlier and later half-periods of MTC patients' postoperative course.

Methods We followed 26 MTC patients (14 hereditary and 12 sporadic MTCs) with postoperative hypercalcitoninemia with periodic measurements of serum calcitonin $(\mathrm{Ct})$ for $>10$ years without major interventions. The median period of $\mathrm{Ct}$ measurements was 18.3 years (range 10.6-30.2 years). We divided the individual patients' study periods into the earlier and later halves and calculated the Ct-DTs for both periods.

Results In the hereditary group, the Ct-DT in the later half-period (Later-Ct-DT) was significantly longer than that in the earlier half-period (Earlier-Ct-DT) (median 20.0 years vs. 7.1 years, $p=0.013$ ). These values in the sporadic group were 20.0 years versus 11.1 years, respectively $(p=0.774)$. Twelve patients (seven hereditary and five sporadic) had Later-Ct-DTs significantly longer than their Earlier-Ct-DTs (median 27.4 years vs. 4.9 years) and good prognoses. Two patients (one hereditary, one sporadic) had Later-Ct-DTs significantly shorter than their Earlier-CtDTs, and both developed structural recurrence and died of the disease.

Conclusion Many of the hereditary and some of the sporadic MTC patients had elongated Ct-DTs over a long period, suggesting spontaneous deceleration and regression of tumor growth. A minority of the MTC patients showed Ct-DT shortening, suggesting tumor growth acceleration.
\end{abstract}

\section{Introduction}

Medullary thyroid carcinoma (MTC) is a rather rare malignancy arising from calcitonin-producing C-cells of the thyroid $[1,2]$. MTCs may arise as a component of multiple endocrine neoplasia type 2A (MEN 2A), type 2B

Akira Miyauchi

miyauchi@kuma-h.or.jp

1 Department of Surgery, Kuma Hospital, 8-2-35 Shimoyamate-dori, Chuo-ku, Kobe 650-0011, Japan

2 Department of Internal Medicine, Kuma Hospital, Kobe, Japan
(MEN 2B), or familial MTC (FMTC). Patients with these hereditary syndromes carry activating germ line mutations of the RET proto-oncogene [3, 4]. MTCs may also occur as sporadic non-hereditary tumors. MTCs secrete calcitonin, and the vast majority of MTCs also produce carcinoembryonic antigen (CEA) [5, 6], the reason for which is not yet known. Calcitonin and CEA serve as very good serum tumor markers of MTCs.

MTCs tend to spread to the regional lymph nodes and distant organs. Compared to sporadic MTCs, hereditary MTCs tend to arise at younger ages [2, 7]. Nodal metastases and postoperative hypercalcitoninemia indicating biochemically persistent disease are common even in 
young patients with hereditary MTCs. The MTCs in MEN $2 \mathrm{~B}$ have an aggressive nature, and their prognosis is generally poor [8]. The prognosis of hereditary MTC in MEN $2 \mathrm{~A}$ and FMTC, however, is generally good [2, 7].

We observed and reported in 1984 that the changes in serum calcitonin levels over time in patients with MTCs who had persistent hypercalcitoninemia postoperatively were exponential and that the calcitonin doubling time (CtDT) was a strong prognostic indicator [9]. Other researchers confirmed that the Ct-DT and the CEA-DT are very strong prognostic indicators of MTCs [10, 11]. Collins et al. [12] studied the changes in the tumor volumes of pulmonary metastases over time and proposed the concept that the growth of human tumors is exponential. The changes in serum calcitonin and CEA fit quite well with this concept $[9,13]$.

It is well known that over a long-term surveillance, the biological nature of some less-aggressive tumors changes to a more aggressive nature, typically from well-differentiated papillary or follicular thyroid carcinoma to poorly differentiated carcinoma or anaplastic thyroid carcinoma [14]. However, a similar change in MTC is not well documented. Patients with MEN 2A or FMTC, on the other hand, often show the following interesting and contradictory phenomena: onsets at young ages, frequent nodal metastases, persistent hypercalcitoninemia postoperatively indicating persistent disease, but relatively good survival and long-term survival even in patients with distant metastases, as we describe below in Materials and methods section. In light of these interesting and contradictory phenomena, we hypothesized that the growth of hereditary MTCs might slow down with aging and that a minority of MTCs acquire an aggressive change over a long term.

To test our hypothesis, we calculated the Ct-DTs in the earlier and later half-periods of the surveillance in MTC patients with postoperative hypercalcitoninemia.

\section{Materials and methods}

\section{Case presentation}

\section{Patient A: an 18-year-old woman with MEN 2A}

In November 1969, an 18-year-old woman underwent a subtotal thyroidectomy with a right modified neck dissection for bilateral thyroid cancers. The pathological diagnosis was bilateral medullary thyroid carcinomas with multiple nodal metastases. In 1975, when the measurement of calcitonin became available, she was found to have hypercalcitoninemia indicating persistent disease (Fig. 1). However, imaging studies failed to reveal metastatic lesions. In 1984, she underwent a bilateral total adrenalectomy for bilateral pheochromocytoma through an abdominal approach. Multiple small nodules were found on her liver surface, a biopsy of which showed metastatic medullary carcinoma. In 1987, she underwent a completion thyroidectomy and left modified neck dissection and extirpation of an enlarged parathyroid gland for recurrent MTC and the appearance of primary hyperparathyroidism. In 2014, at 63 years old, she was asymptomatic with elevated serum calcitonin $(3900 \mathrm{pg} / \mathrm{ml})$ and CEA $(177 \mathrm{ng} / \mathrm{ml})$ levels. Imaging studies revealed multiple small low-density lesions with spotty calcifications in her liver, consistent with multiple small liver metastases. She carries RET codon 634 mutation.

The changes in her serum calcitonin levels over time and major clinical events are shown in Fig. 1. Periodic measurements of serum calcitonin following the initial thyroid surgery showed a moderate increase in the values with the Ct-DT of 8.7 years, a decrease after the second neck surgery followed by a similar increase with Ct-DT of 9.1 years, and interestingly, a significant gradual decrease in serum calcitonin levels, giving a negative value to $\mathrm{Ct}$ DT at -63.0 years since approx. 45-50 years of age, without any causative events.

\section{Methods}

From April 1969 to April 2005, 175 patients with MTC underwent surgical treatment at Kuma Hospital. Among them, patients who satisfied the following inclusion criteria were selected for the present study: patients who had obvious persistent hypercalcitoninemia postoperatively, were followed for $>10$ years following the initial surgery or the redo surgery if this was performed, and did not receive major interventions such as surgery or external beam radiation during the study period. Tyrosine kinase inhibitors for medullary thyroid carcinoma were not available in the present study period in Japan and were not used for the present patients.

There were 26 patients (21 females and five males) (Table 1). Fourteen had hereditary MTC (nine MEN 2A and five FMTC). Twelve patients had sporadic MTC. All 14 of the patients with hereditary MTC carried germ line RET mutations at codons $634,620,618,611$, and 768 in 8 , $2,1,1$, and 2 patients, respectively, and none of the 12 patients with sporadic MTC was found to carry the mutations (Table 1). Two of the hereditary MTC patients underwent their initial thyroid surgery elsewhere. The extent of disease progression and surgery performed for these patients are shown in Table 2. Majority but not all of the present patients had rather advanced disease such as stage IVA. Therefore, they underwent rather extensive surgery. Seven of the hereditary MTC patients and two of 
Fig. 1 Changes in serum calcitonin levels and major clinical events in Patient A with MEN 2A. She underwent the first thyroid surgery at 18 years old for MTC, a bilateral adrenalectomy at 33 years old, and the second neck surgery for recurrent tumor at 36 years old. Note that the vertical axis for calcitonin values is shown in $\log$ scale. Lines show the regression lines in three periods: before the redo surgery, the earlier halfperiod, and the later half-period after redo surgery. Redo Op.: the second neck surgery for recurrent tumor. Adx: bilateral adrenalectomy for bilateral pheochromocytoma. Numbers indicate Ct-DT values (years)

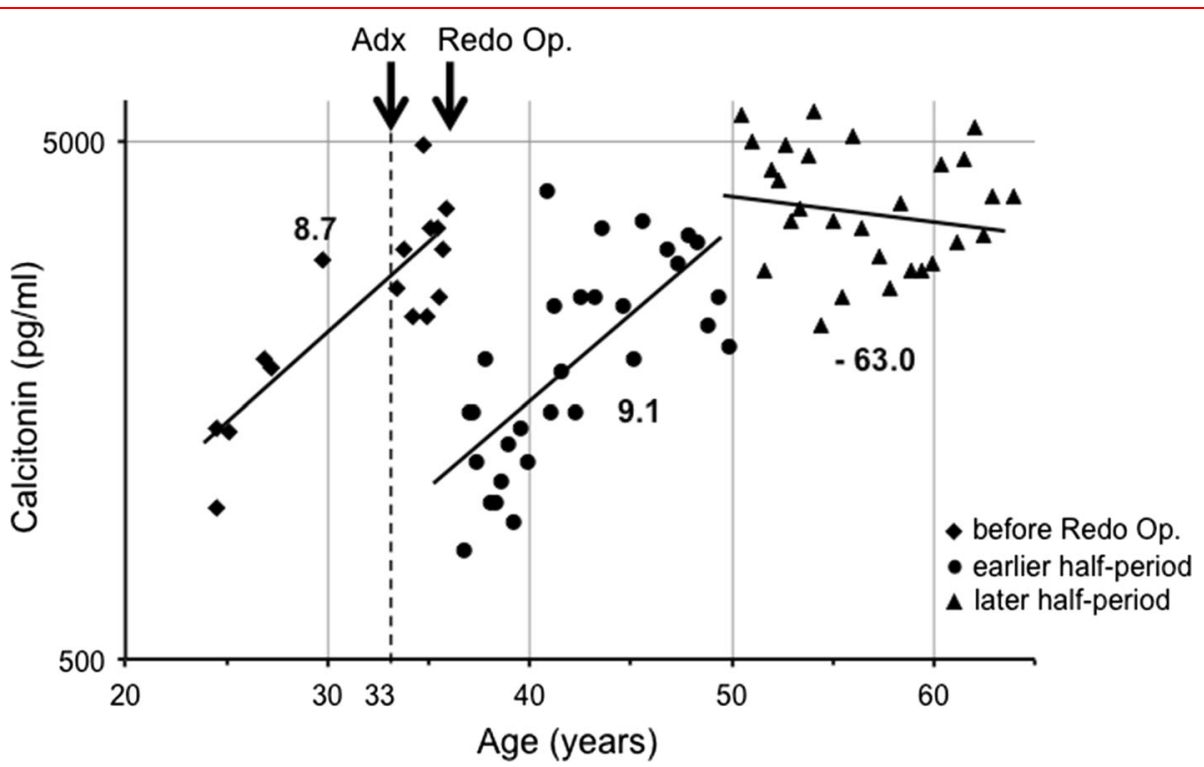

Table 1 Clinical features of the 26 patients with medullary thyroid carcinoma (MTC)

\begin{tabular}{|c|c|c|c|}
\hline & Hereditary MTC & Sporadic MTC & Significance \\
\hline No. of patients, female: male & $14(12: 2)$ & $12(9: 3)$ & $P=0.490$ \\
\hline Primary op.: Redo op. & $12: 2$ & $12: 0$ & $P=0.173$ \\
\hline Phenotype: MEN 2A: MTC only & $9: 5$ & $0: 12$ & $P=0.001$ \\
\hline Redo op. before the study period & 7 & 2 & $P=0.075$ \\
\hline Age at first surgery (years) ${ }^{\mathrm{a}}$ & $38(18-76)$ & $40.5(32-66)$ & $P=0.227$ \\
\hline Max. tumor size $(\mathrm{mm})^{\mathrm{a}}$ & $25(14-50)$ & $26.5(13-50)$ & $P=0.543$ \\
\hline Follow-up period $(\mathrm{yrs})^{\mathrm{a}}$ & $28.4(10.9-45.1)$ & $21.1(10.6-28.4)$ & $P=0.123$ \\
\hline $\mathrm{Ct}$ follow-up period (years) ${ }^{\mathrm{a}}$ & $19.1(10.8-30.2)$ & $17.5(10.6-28.4)$ & $P=0.718$ \\
\hline No. of $\mathrm{Ct}$ measurements $\mathrm{s}^{\mathrm{a}}$ & $29.5(10-77)$ & $40(17-91)$ & $P=0.268$ \\
\hline Died of disease & 1 & 2 & $P=0.449$ \\
\hline Died of other disease & 1 & 1 & $P=0.910$ \\
\hline
\end{tabular}

${ }^{a}$ Median (range). Ct, calcitonin; MEN 2A, multiple endocrine neoplasia type 2A; MTC, medullary thyroid carcinoma

the sporadic MTC patients had redo surgery for recurrent disease before the present study period. None of the patients had structural disease detected on imaging studies at the beginning of the present study period except for Patient A described above.

The median surveillance periods for the hereditary MTC patients and the sporadic MTC patients were 28.4 years and 21.1 years, respectively. Two patients had redo surgery for recurrent disease after the study period, and three died of the disease.

Patients were seen typically twice a year with measurements of serum calcitonin, and imaging studies were added when indicated. Serum calcitonin was measured for each visit by a laboratory (SRL, Tokyo) using the solid two-site immunoradiometric assay (Mitsubishi Chemical,
Tokyo). This was the only approved measurement method for calcitonin in Japan until April 2015, and the end of the present study period was set at that time.

The median Ct follow-up periods in the hereditary MTC and sporadic MTC patients were 19.1 years and 17.5 years, respectively (Table 1). The two groups' median numbers of calcitonin measurements were 29.5 and 40 , respectively. We divided each patient's study period into the earlier and later halves and calculated the calcitonin doubling time (Ct-DT) for each period as described [9]. In brief, we calculated a regression line from the changes in $\mathrm{Ct}$ values over time for each period in individual patients. Then, CtDT was calculated with the slope $(b)$ of the regression line as $(\log 2) / b$. 
Table 2 TNM stage and the initial surgery performed for the 26 patients with medullary thyroid carcinoma (MTC)

\begin{tabular}{|c|c|c|c|}
\hline & Hereditary MTC & Sporadic MTC & Significance \\
\hline TNM stage & & & $P=0.817$ \\
\hline T1bN0M0 stage I & 1 & 1 & \\
\hline T2NOM0 stage II & 4 & 5 & \\
\hline T1bN1bM0 stage IVA & 0 & 1 & \\
\hline T2N1bM0 stage IVA & 5 & 4 & \\
\hline T3N1bM0 stage IVA & 2 & 1 & \\
\hline Unknown & 2 & 0 & \\
\hline Surgery & & & $P=0.082$ \\
\hline Hemi Tx & 0 & 1 & \\
\hline Hemi Tx $+M$ & 0 & 3 & \\
\hline Subtotal Tx $+M$ & 3 & 0 & \\
\hline Total Tx $+\mathrm{M}$ & 4 & 2 & \\
\hline Total Tx + M2 & 5 & 4 & \\
\hline Total Tx $+\mathrm{M}+$ Med & 0 & 2 & \\
\hline Unknown & 2 & 0 & \\
\hline
\end{tabular}

TNM stage according to UICC eighth edition. Hemi Tx: hemithyroidectomy, Subtotal Tx: subtotal thyroidectomy, Total Tx: total thyroidectomy, M: modified neck dissection, M2: bilateral modified neck dissection, and Med: mediastinal dissection. N.S.: not significant

\section{Statistical analyses}

The statistical tests used to compare groups included Student's $t$ test, the Mann-Whitney $U$-test for skewed variables, and the Chi-square test for differences in count and frequency. As described below in Results section, some of the patients showed a decrease in serum calcitonin levels over time, giving negative values for Ct-DTs. This created a discontinuity problem between the positive Ct-DT values and the Ct-DTs in negative values. Thus, the statistical analyses on DTs were performed on the reciprocal of DT (i.e., 1/DT) as Barbet et al. described [10]. Ct-DT was calculated from the slope of the regression line of the changes in $\mathrm{Ct}$ values over time for the earlier and later halfperiods in individual patients. We evaluated the difference between the slope of the regression line in the earlier halfperiod and that in the later half-period for each individual patient by using a standardized major axis (SMA) technique [15]. If the difference in these slopes in a patient was significant, the difference in the Earlier-Ct-DT value and the Later-Ct-DT value in the patient was regarded significant. Statistical analyses were performed using the program Stat Flex (View Flex, Tokyo). A $p$ value $<0.05$ was considered significant.

\section{Results}

Many of the present patients showed a decrease in the increase rate of their serum calcitonin level over a long period; some showed a decrease in their calcitonin levels, whereas some showed no change in the increase rate, and a few showed a sudden increase in the levels. Representative cases are shown in Figs. 1 and $2 \mathrm{a}-\mathrm{d}$.

Among the 14 hereditary MTC patients and in the earlier half-period of the study period, 13 patients showed a gradual increase in their serum calcitonin levels with the median Ct-DT of 7.2 years, and the other hereditary MTC patient showed a gradual decrease in the level with the CtDT of -7.6 years. In the later half-period, 12 showed a slower increase in their serum calcitonin levels with the median Ct-DT of 15.7 years, and the other two patients showed a decrease in the levels with the median Ct-DT of -62.2 years (Table 2). In this group of hereditary MTC patients, the Later-Ct-DT (median, 20.0 years) was significantly longer than the Earlier-Ct-DT (median, 7.1 years) ( $p=0.013$ ) (Table 3, Fig. 3), indicating that the growth rate in the later half-period was decreased compared to that in the earlier half-period. However, two of the hereditary MTC patients showed shortening in their Ct-DT indicating an increase in their tumor growth rate, as demonstrated in Fig. 3.

Among the 12 sporadic MTC patients and in the earlier half-period of the surveillance, ten patients showed a gradual increase in their serum calcitonin levels with the median Ct-DT of 8.0 years, and the other two patients showed a gradual decrease in these levels with the median Ct-DT of -22.9 years. In the later half-period, nine patients showed a slower increase with the median Ct-DT of 12.0 years, and the other three patients showed a decrease with the median Ct-DT of -31.5 years (Table 4 ). In the group of sporadic MTC patients, the Later-Ct-DT 
(a)

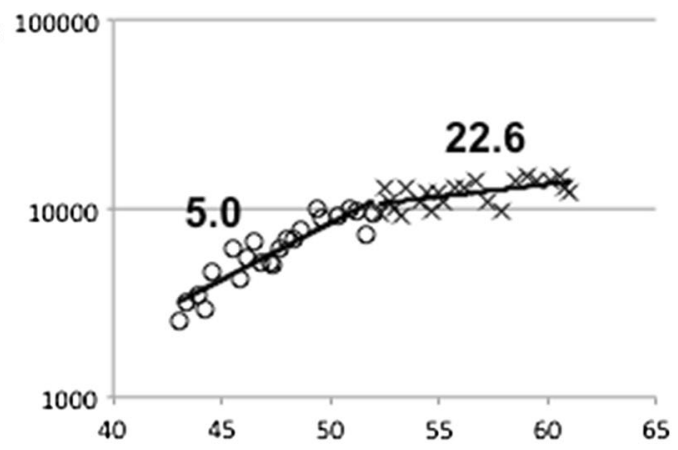

(c)

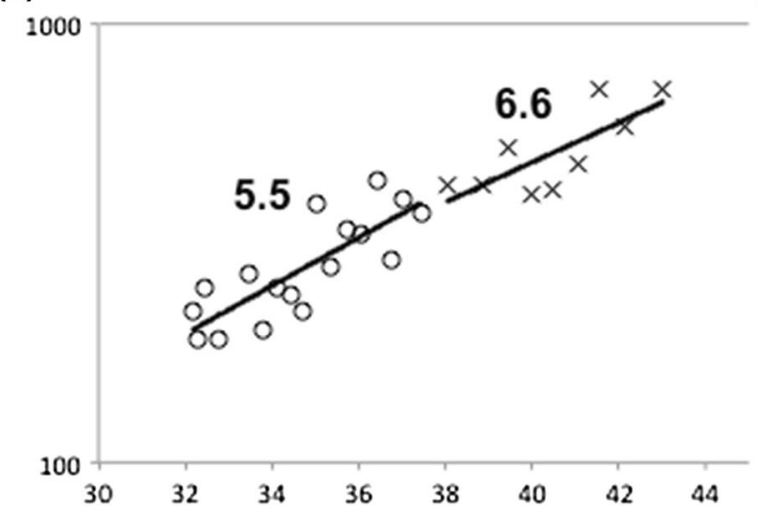

(b)

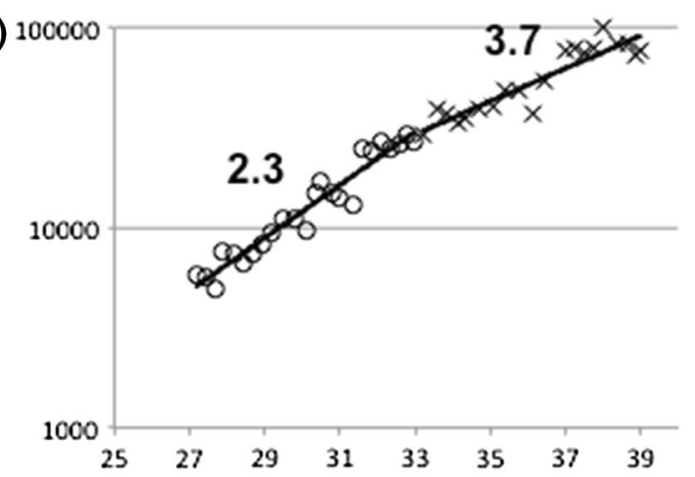

(d)

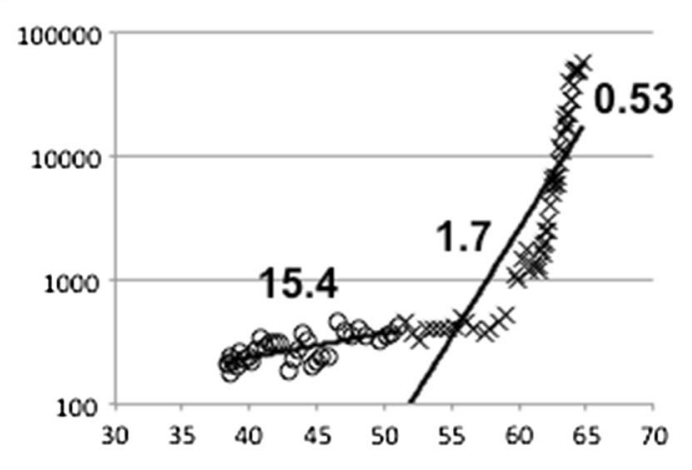

Fig. 2 Representative cases showing changes in serum calcitonin levels over long periods. a A patient who had a significantly longer Later-CtDT $(22.6$ years) than Earlier-Ct-DT $(4.0$ years $)(p<0.01)$. b A patient who had a significantly longer Later-Ct-DT $(3.7$ years $)$ than Earlier-CtDT (2.3 years) $(p<0.01)$. c A patient whose Later-Ct-DT (6.6 years) and Earlier-Ct-DT (5.5 years) were not significantly different (n.s.). d A patient who had a significantly shorter Later-Ct-DT (1.7 years) than Earlier-Ct-DT (15.4 years) $(p<0.01)$. The Ct-DT after the aggressive change was 0.53 years. The vertical axes indicate the serum calcitonin levels (pg/ml) shown on $\log$ scale. The horizontal axes indicate the patient's age (years). Lines indicate regression lines. Numbers indicate Ct-DT (years)

Table 3 Calcitonin doubling time (Ct-DT) in the earlier and later half-periods in the 14 patients with hereditary MTC

\begin{tabular}{|c|c|c|c|}
\hline & \multicolumn{2}{|l|}{ Ct-DT } & \multirow[t]{2}{*}{ Significance } \\
\hline & Earlier period & Later period & \\
\hline Surveillance period (years) & $9.4(5.2$ to 14.3$)(n=14)$ & $8.9(1.1$ to 14.7$)(n=14)$ & $P=0.748$ \\
\hline No. of measurements & $19(4-38)(n=14)$ & $13.5(5-39)(n=14)$ & $P=0.250$ \\
\hline \multicolumn{4}{|l|}{ Doubling time (years) } \\
\hline Positive value & $7.2(2.3-117.9)(n=13)$ & $15.7(1.2-333.0)(n=12)$ & - \\
\hline Negative value & $-7.6(-7.6)(n=1)$ & $-62.2(-63.3$ to -61.0$)(n=2)$ & - \\
\hline $1 /$ doubling time (1/years) & $0.14(-0.13$ to 0.44$)(n=14)$ & $0.05(-0.02$ to 0.81$)(n=14)$ & $P=0.013$ \\
\hline Median DT converted from the median 1/DT (years) & 7.1 & 20.0 & - \\
\hline
\end{tabular}

-: not applicable. Values are medium (range)

(median, 20.0 years) was longer than the Earlier-Ct-DT (median, 11.1 years); however, the difference did not reach significance (Table 4, Fig. 3). Although there was no significant difference as a group, many of the sporadic MTC patients showed elongation of Ct-DT, whereas some of them showed shortening of Ct-DT, suggesting a decrease and increase in their tumor growth rate, respectively (Fig. 3).

When the patients were grouped according to the length of the surveillance, among the 15 patients who had 10-20 years surveillance their Later-Ct-DTs were not significantly different from their Earlier-Ct-DTs as a group: 
Fig. 3 Reciprocals of the CtDTs in the earlier and later halfperiods in the patients with hereditary MTC and sporadic MTC. The medians (range) for these values are given in Tables 2 and 3

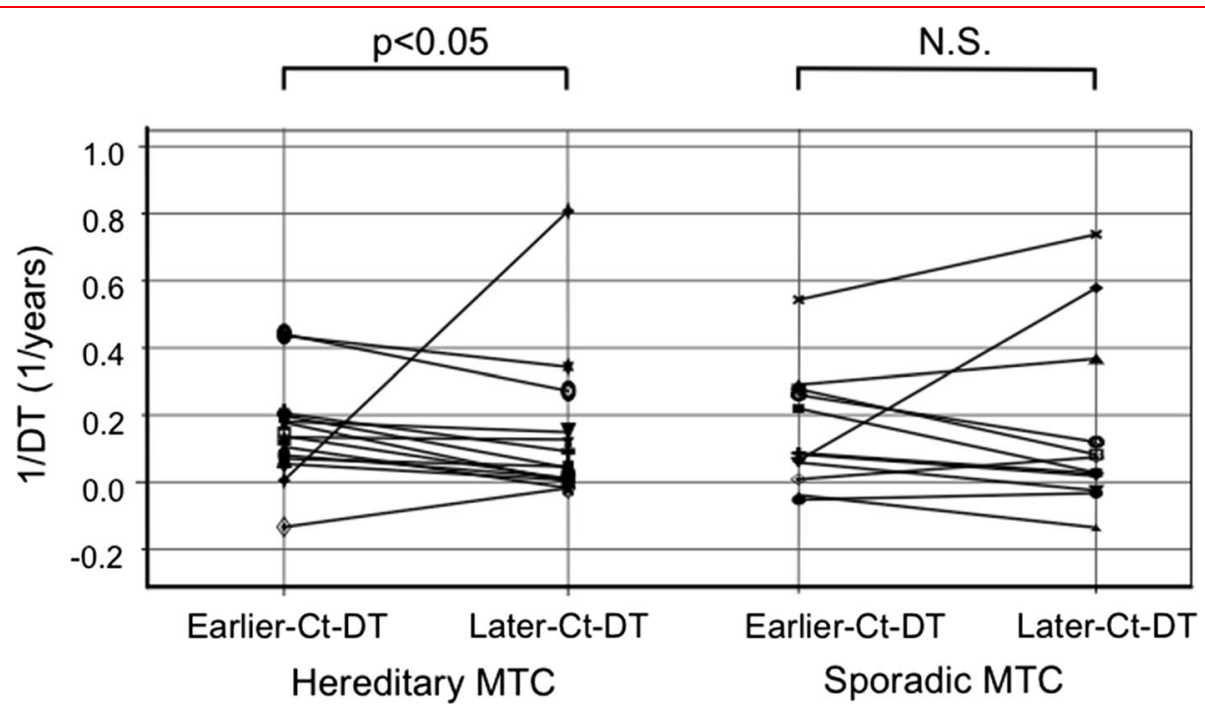

Table 4 Calcitonin doubling time (Ct-DT) in the earlier and later half-periods in patients with sporadic medullary thyroid carcinoma

\begin{tabular}{|c|c|c|c|}
\hline & \multicolumn{2}{|l|}{ Ct-DT } & \multirow[t]{2}{*}{ Significance } \\
\hline & Earlier period & Later period & \\
\hline Surveillance period (years) & $7.5(2.9-13.8)(n=12)$ & $7.6(5.1-13.5)(n=12)$ & $P=0.686$ \\
\hline No. of measurements & $18(10-38)(n=12)$ & $18.5(6-57)(n=12)$ & $P=0.795$ \\
\hline \multicolumn{4}{|l|}{ Doubling time (years): } \\
\hline Positive value & $8.0(1.8-114.3)(n=10)$ & $12.0(1.4-51.8)(n=9)$ & - \\
\hline Negative value & $-22.9(-26.5$ to -19.3$)(n=2)$ & $-31.5(-41.0$ to -7.4$)(n=3)$ & - \\
\hline 1/doubling time (1/years) & $0.09(-0.05$ to 0.54$)(n=12)$ & $0.05(-0.14$ to 0.74$)(n=12)$ & $P=0.774$ \\
\hline Median DT converted from the median 1/DT (years) & 11.1 & 20.0 & - \\
\hline
\end{tabular}

Values are medium (ranges)

median (ranges) of 1/DT being $0.075(-0.135-0.738)$ and 0.139 (-0.131-0.543), respectively $(p=0.302)$ (Fig. 4a). While the analyses on the difference between the slope of the regression line of the changes in $\mathrm{Ct}$ values over time in the earlier half-period and that in the later half-period in individual patients showed that six of them had significantly longer Later-Ct-DT than his or her Earlier-Ct-DT, indicating deceleration and regression of tumor growth in these patients. Among the 11 patients with $>20$ years surveillance, their Later-Ct-DTs were not significantly different from their Earlier-Ct-DTs $(p=0.065)$ as a group, probably because of the presence of two patients with markedly shortened Later-Ct-DT. The remaining patients showed elongation in Later-Ct-DT (Fig. 4b).

Our analysis on the difference in the slopes of the regression lines of the changes in $\mathrm{Ct}$ values over time in these two periods in individual patients revealed that 12 patients (seven hereditary MTC patients and five sporadic MTC patients) had significantly longer Later-Ct-DTs compared to their Earlier-Ct-DTs (median Ct-DT: 27.4 vs. 4.9 years). Of these 12 patients, five hereditary MTC patients and five sporadic MTC patients had a Later-Ct-DT that was more than two times the value of their Earlier-CtDT, indicating a significant slowdown in the growth rate. On the other hand, two patients (one hereditary MTC patient and one sporadic MTC patients) had a significantly and markedly shorter Later-Ct-DT than Earlier-Ct-DT (1.2 vs. 125 years and 1.7 vs. 15.4 years, respectively) (Figs. 2d, 4b). These data indicate that 12 of the present patients had a decrease in their tumor growth rate over a long period; that is, "spontaneous deceleration of tumor growth.' Three of these patients had negative Later-Ct-DT values indicating 'regression of the tumor,' whereas two patients had a marked shortening in their Ct-DT that might indicate 'acceleration of tumor growth' reflecting an 'aggressive change' in the nature of their tumors. These two patients developed structural recurrence in bones and lungs and died of the disease. 

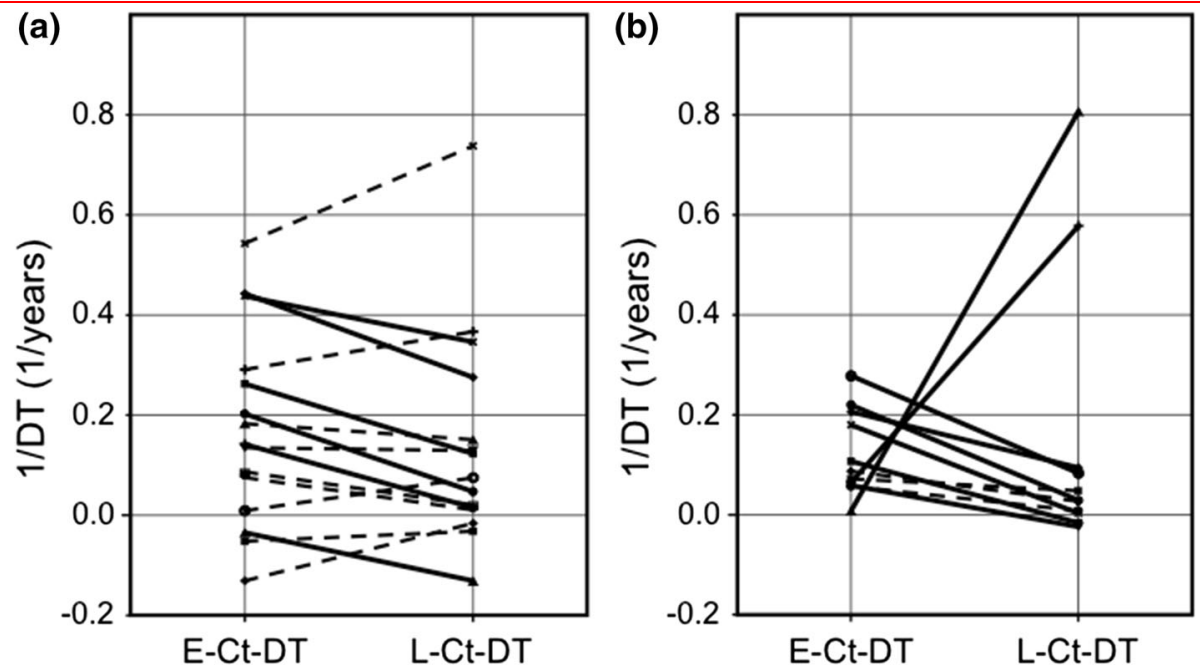

Fig. 4 Reciprocals of Ct-DTs in the earlier and later half-periods in the patients with MTC. a (left panel): Patients who had 10-20 years surveillance. b (right panel): Patients who had $>20$ years surveillance. Fourteen patients who had significant differences between their Ct-DTs in the earlier and later half-periods are shown with thick lines, and 12 patients without significant differences are shown with broken lines. Eight of the 11 patients with $>20$ years surveillance had significant differences in these two values, while six of the 15 patients with 10-20 years surveillance had significant differences in these two values. Twelve of these 14 patients with significant differences in their earlier and later half-period values had later half-period 1/DT values smaller than their earlier half-period 1/DT values, indicating deceleration in their tumor growth rates, while the remaining two patients had later half-period 1/DT values larger than their earlier half-period 1/DT values indicating acceleration in their tumor growth

\section{Discussion}

Our present findings revealed that the majority of the 14 patients with hereditary MTC who had persistent hypercalcitoninemia postoperatively showed elongation of their Ct-DTs over a long period, indicating deceleration in their tumor growth rate. Two patients showed a decrease in their serum calcitonin levels, indicating possible tumor regression. Change in serum tumor marker levels over time in a particular patient is generally regarded as a surrogate for the change in tumor mass of the patient, and the change is best expressed as serum tumor marker-doubling time. Elongation of serum tumor marker-doubling time may indicate slowing of tumor growth rate, or alternatively it may be due to decreasing production of the tumor marker by the tumor that has a constant growth. Without tumor mass data, one cannot distinguish between these two possibilities. We could not evaluate the tumor volume-doubling times, since most of the present MTC patients did not have measurable structural diseases. However, the patients with elongation of their Ct-DTs had good prognoses, and none of them died of the disease during the long-term surveillance. In addition, patients who had shortening of their Ct-DT developed structural recurrence and died of MTC.

The changes between the Earlier-Ct-DT and the LaterCt-DT in the present 12 sporadic MTC patients did not reach significance. However, this was due to the presence of some patients who had a shorter Later-Ct-DT than Earlier-Ct-DT. Five of the 12 sporadic MTC patients did have significantly longer Later-Ct-DTs than Earlier-CtDTs, indicating that spontaneous deceleration of tumor growth occurred in sporadic MTCs too.

On the other hand, one patient with hereditary MTC and another patient with sporadic MTC showed shortening of their Ct-DT and both of them developed structural recurrence and died of the disease, consistent with aggressive change in their tumor nature.

Although the precise underlying mechanisms have not been established, the phenomena of spontaneous regression have been well documented in many malignant tumors such as neuroblastoma, hypernephroma, malignant melanoma, choriocarcinoma, and malignant lymphoma [16, 17]. Thus, spontaneous tumor regression and a growth deceleration might not be rare phenomena.

Mazzaferri et al. [18] reported that papillary thyroid carcinomas (PTCs) showed a unique biological behavior related to the patient's age at surgery. Young patients showed high recurrence rates but excellent survival, and elderly patients showed high recurrence rates and high mortality rates compared to middle-aged patients [18]. These confusing agerelated phenomena are not yet understood. In our study of 426 PTC patients who underwent a total thyroidectomy, the proportion of patients with detectable serum thyroglobulin indicating biochemically persistent disease was high in the patients aged $<40$ years and those $\geq 60$ years and low in the 
patients 40-60 years. The proportion of patients with a short thyroglobulin-DT and a high Ki-67 labeling index indicating rapid tumor growth increased with age, and the proportion of PTC patients with a long thyroglobulin-DT and a low Ki-67 labeling index indicating slow tumor growth decreased with age $[19,20]$. Thus, the growth of PTCs is strongly related to age. However, these were cross-sectional observations.

Here, we report, for the first time, a spontaneous deceleration of tumor growth in hereditary MTCs based on long-term longitudinal observations of the same patients. Some of the sporadic MTCs also showed a similar phenomenon. A similar spontaneous growth deceleration might occur in the PTCs of young patients; this may be demonstrated with careful longitudinal observations. If this is the case, the confusing phenomena in which PTCs in children often advance and show high recurrence rates and yet the patients show excellent survival can be understood, although the underlying mechanisms remain to be clarified.

Gain of function germ line and somatic mutations in $R E T$ proto-oncogene were reported as pathogenetic mutations of hereditary MTC and sporadic MTC, respectively $[3,4]$. RET/PTC rearrangements and BRAF mutations were also reported in PTC [21-23]. All of these mutations are involved in the MAPK signaling pathway and activate the pathway. Age might be an important factor affecting this pathway through as-yet unknown mechanisms.

Two of the present 26 MTC patients (one hereditary and one sporadic) showed a marked shortening in their Ct-DTs over time indicating an aggressive change in their tumors' nature, and they developed structural recurrence and died of the disease. Some MTCs may have aggressive changes over a long-term period similar to the poor differentiation or anaplastic change in differentiated thyroid cancer.

Our present analyses revealed spontaneous deceleration and possible regression in tumor growth in hereditary MTCs as well as in some of the sporadic MTCs, and aggressive change in a few of the MTCs. However, this patient series was carefully selected, based on the inclusion criteria with a surveillance period $>10$ years without major interventions. Patients who had recurrence and were treated for it within 10 years were not included. Therefore, the proportions of MTC patients in general who show these changes in tumor growth rates remain to be clarified. Our findings must therefore be tested in future studies, and the mechanisms underlying these phenomena also remain to be elucidated.

\section{Conclusion}

Many of the hereditary and some of the sporadic MTC patients had elongated Ct-DTs over a long period, suggesting spontaneous deceleration and regression of tumor growth. A minority of the MTC patients showed Ct-DT shortening, suggesting an aggressive change in their tumor nature.

Acknowledgements To solve the problems encountered in the calculation of Ct-DTs and tumor volume-DTs, we created the 'Doubling Time and Progression Calculator.' This can be downloaded at Kuma Hospital's website: http://www.kuma-h.or.jp/english/.

\section{Compliance with ethical standards}

Conflict of interest The authors declare that they have no conflict of interest.

Open Access This article is distributed under the terms of the Creative Commons Attribution 4.0 International License (http://crea tivecommons.org/licenses/by/4.0/), which permits unrestricted use, distribution, and reproduction in any medium, provided you give appropriate credit to the original author(s) and the source, provide a link to the Creative Commons license, and indicate if changes were made.

\section{References}

1. Ito Y, Miyauchi A, Yabuta T et al (2009) Alternative surgical strategies and favorable outcomes in patients with medullary thyroid carcinoma in Japan: experience of a single institution. World J Surg 33:58-66. https://doi.org/10.1007/s00268-0089795-2

2. Moley JF, Fialkowski EA (2007) Evidence-based approach to the management of sporadic medullary thyroid carcinoma. World $\mathbf{J}$ Surg 31:946-956. https://doi.org/10.1007/s00268-006-0846-2

3. Mulligan LM, Kwok JB, Healey CS et al (1993) Germ-line mutations of the RET proto-oncogene in multiple endocrine neoplasia type 2A. Nature 363:458-460

4. Eng C, Smith DP, Mulligan LM et al (1994) Point mutation within the tyrosine kinase domain of the RET proto-oncogene in multiple endocrine neoplasia type $2 \mathrm{~B}$ and related sporadic tumours. Hum Mol Genet 3:237-241

5. Melvin KE, Miller HH, Tashjian AH Jr (1971) Early diagnosis of medullary carcinoma of the thyroid gland by means of calcitonin assay. N Engl J Med 285:1115-1120

6. Ishikawa N, Hamada S (1976) Association of medullary carcinoma of the thyroid with carcinoembryonic antigen. Br J Cancer 34:111-115

7. van Heerden JA, Grant CS, Gharib H et al (1990) Long-term course of patients with persistent hypercalcitoninemia after apparent curative primary surgery for medullary thyroid carcinoma. Ann Surg 212:395-400

8. Machens A, Niccoli-Sire P, Hoegel J et al (2003) European multiple endocrine neoplasia (EUROMEN) study group: early malignant progression of hereditary medullary thyroid cancer. N Engl J Med 349:1517-1525

9. Miyauchi A, Onishi T, Morimoto S et al (1984) Relation of doubling time of plasma calcitonin levels to prognosis and recurrence of medullary thyroid carcinoma. Ann Surg 199:461-466

10. Barbet J, Campion L, Kraeber-Bodéré F, GTE Study Group et al (2005) Prognostic impact of serum calcitonin and carcinoembryonic antigen doubling-times in patients with medullary thyroid carcinoma. J Clin Endocrinol Metab 90:6077-6084

11. Laure Giraudet A, Al Ghulzan A, Aupérin A et al (2008) Progression of medullary thyroid carcinoma: assessment with 
calcitonin and carcinoembryonic antigen doubling times. Eur $\mathbf{J}$ Endocrinol 158:239-246

12. Collins VP, Loeffler RK, Tivey H (1956) Observations on growth rates of human tumors. Am J Roentgenol Radium Ther Nucl Med 76:988-1000

13. Miyauchi A, Matsuzuka F, Kuma K et al (1988) Evaluation of surgical results and prediction of prognosis in patients with medullary thyroid carcinoma by analysis of serum calcitonin levels. World J Surg 12:610-615

14. Wiseman SM, Griffith OL, Deen S et al (2007) Identification of molecular markers altered during transformation of differentiated into anaplastic thyroid carcinoma. Arch Surg 142:717-727

15. Warton DI, Duursma RA, Falster DS et al (2012) smart 3: an R package for estimation and inference about allometric lines. Method Ecol Evol 3:257-259

16. Brodeur GM, Bagatell R (2014) Mechanisms of neuroblastoma regression. Nat Rev Clin Oncol 11:704-713

17. Gattiker HH, Wiltshaw E, Galton DA (1980) Spontaneous regression in non-Hodgkin's lymphoma. Cancer 45:2627-2632

18. Mazzaferri EL, Kloos RT (2001) Clinical review 128: current approaches to primary therapy for papillary and follicular thyroid cancer. J Clin Endocrinol Metab 86:1447-1463
19. Miyauchi A, Kudo T, Kihara M et al (2013) Relationship of biochemically persistent disease and thyroglobulin-doubling time to age at surgery in patients with papillary thyroid carcinoma. Endocr J 60:415-421

20. Miyauchi A, Kudo T, Hirokawa M et al (2013) Ki-67 labeling index is a predictor of postoperative persistent disease and cancer growth and a prognostic indicator in papillary thyroid carcinoma. Eur Thyroid J 2:57-64

21. Grieco M, Santoro M, Berlingieri MT et al (1990) PTC is a novel rearranged form of the ret proto-oncogene and is frequently detected in vivo in human thyroid papillary carcinomas. Cell 60:557-563

22. Santoro M, Dathan NA, Berlingieri MT et al (1994) Molecular characterization of RET/PTC3; a novel rearranged version of the RET proto-oncogene in a human thyroid papillary carcinoma. Oncogene 9:509-516

23. Kimura ET, Nikiforova MN, Zhu Z et al (2003) High prevalence of BRAF mutations in thyroid cancer: genetic evidence for constitutive activation of the RET/PTC-RAS-BRAF signaling pathway in papillary thyroid carcinoma. Cancer Res 63:1454-1457 